\title{
Impacto de los cuidados predialíticos en el tiempo hasta el inicio de diálisis en una cohorte de pacientes con enfermedad renal crónica en Colombia
}

\author{
Ángela S. Rivera1*, Delia Perea², Ricardo Sánchez³ ${ }^{3}$ Kindar R. Astudillo², J. Mauricio Uribe², \\ Izcay Ronderos², Luis F. Moreno ${ }^{2}$, Alfonso Bunch², Leyder Corzo ${ }^{2}$ y Mauricio Sanabria² \\ ${ }^{1}$ División Renal, Baxter Latinoamérica; ${ }^{2}$ Renal Therapy Services; ${ }^{3}$ Universidad Nacional de Colombia. Bogotá, Colombia
}

\section{Resumen}

Introducción: El cuidado predialítico de los pacientes con enfermedad renal crónica (ERC) puede mejorar su estado clínico y postergar el inicio de la terapia de reemplazo renal (TRR). Objetivo: Establecer si los cuidados predialíticos únicamente con un nefrólogo o con un equipo multidisciplinario prolongan el tiempo hasta el inicio de la diálisis y reducen el riesgo de un inicio urgente de la TRR en pacientes colombianos. Materiales y métodos: Estudio de cohorte retrospectiva en el que se incluyeron pacientes mayores de 18 años, con diagnóstico de ERC, con una tasa de filtración glomerular estimada, según el método de Cockcroft-Gault, menor a $20 \mathrm{ml} / \mathrm{min}$ al comienzo del seguimiento. El periodo de reclutamiento de la cohorte estuvo comprendido entre el 1 junio de 2008 y el 31 marzo de 2010. Para el análisis se utilizó estadística descriptiva, modelos multivariados generalizados $\gamma$ para evaluar el riesgo de inicio de diálisis y análisis de regresión logística para determinar las probabilidades de que los pacientes requirieran un inicio urgente de la TRR. Resultados: Se evaluaron 769 pacientes. El $12.1 \%$ recibió cuidados predialíticos multidisciplinarios; el 42.1\%, cuidados únicamente del nefrólogo, y el 45.8\% de los pacientes no los recibieron. Aquellos pacientes a quienes se les administraron cuidados predialíticos, ya fuera multidisciplinarios o únicamente del nefrólogo, presentaron un riesgo significativamente menor de inicio urgente de la diálisis que aquéllos que no habían recibido intervención alguna. Conclusión: El cuidado provisto por un equipo multidisciplinario o por el nefrólogo aumenta el tiempo libre de diálisis en pacientes con ERC avanzada.

Palabras clave: Hemodiálisis. Diálisis peritoneal. Grupo de atención al paciente. Enfermedad renal crónica.

\section{Impact of pre-dialytic care over time to the start of dialysis in a cohort of patients with} chronic kidney disease in Colombia

\section{Abstract}

Background: Predialysis care of patients with chronic kidney disease (CKD) may improve their clinical condition and delay initiation of renal replacement therapy (RRT). Objective: To determine whether predialysis care from a nephrologist alone or with a multidisciplinary team lengthens the time to RRT and reduces the risk of an urgent start of RRT among patients in Colombia. Materials and methods: A design of retrospective cohort was used, included older than 18 years, with chronic

\section{Correspondencia:}

*Ángela S. Rivera

E-mail: angela_rivera@baxter.com
Disponible en internet: 28-05-2018 Nefro Lat. 2018;15:8-15 www.nefrologialatinoamericana.com 2444-9032/@ 2018 Sociedad Latinoamericana de Nefrología e Hipertensión. Publicado por Permanyer México SA de CV. Este es un artículo Open Access bajo la licencia CC BY-NC-ND (http://creativecommons.org/licenses/by-nc-nd/4.0/). 
kidney disease (CKD) estimated glomerular filtration rate according to the Cockcroft-Gault method at $<20 \mathrm{~mL} / \mathrm{min}$ at the beginning of follow-up, recruitment in the cohort was between June 1, 2008 and March 31, 2010. For the analysis, we used descriptive statistics, univariate and multivariate generalized gamma analyses were performed to assess the risk for initiating $R R T$ over time. A logistic regression analysis was conducted to determine the odds for patients to require the start of RRT in an urgent situation. Results: 769 patients were included. Just $12.1 \%$ of the patients had multidisciplinary predialysis care, $42.1 \%$ had nephrology-only care and $45.8 \%$ of patients received no predialysis intervention. Median time to RRT initiation was 5.2 months. Patients who received predialysis multidisciplinary or nephrology-only care had a significantly lower risk of an urgent-start dialysis than those who had no prior intervention. Conclusion: Care provided by a multidisciplinary team or by the nephrologist increases dialysis free time in patients with advanced chronic kidney disease.

Key words: Hemodialysis. Patient care team. Peritoneal dialysis. Chronic kidney diseases.

\section{Introducción}

La ERC es un importante problema de salud pública debido al aumento de su incidencia y prevalencia, y a los costos relacionados con su tratamiento ${ }^{1-3}$. Los sistemas de salud y las sociedades científicas están desarrollando procedimientos y directrices multidisciplinarios para controlar la morbilidad, la mortalidad y la progresión de la ERC, impedir o aplazar la TRR, o preparar a los pacientes para la TRR, en caso de que sea necesaria.

A pesar de que los beneficios de los programas de prevención están bien documentados ${ }^{4}$, en muchos países los pacientes con ERC no son remitidos a los nefrólogos oportunamente, con lo que se pierde así la oportunidad de controlar la morbilidad, retrasar el avance de la enfermedad, planear el inicio de la TRR y mejorar la calidad de vida relacionada con la salud general de los pacientes ${ }^{1-3,5-7}$. Aquellos pacientes que, en el curso de su enfermedad, son remitidos tardíamente a un nefrólogo o a un equipo multidisciplinario son más propensos a requerir un inicio intempestivo de la TRR, así como a presentar tasas más elevadas de hospitalización y de mortalidad, y, por consiguiente, aumentan el consumo de recursos destinados a la salud y los costos ${ }^{5-8}$.

Muchos grupos de nefrólogos en el mundo están implementando programas de prevención con cuidados multidisciplinarios para preparar a los pacientes para la TRR, pero aún no ha sido validado en su totalidad el beneficio completo de estos programas para los pacientes con $\mathrm{ERC}^{7,9}$.

Las recomendaciones del grupo Kidney Disease Improving Global Outcomes (KDIGO), en el informe de 2012 sobre la ERC, establecen que los pacientes con ERC progresiva deben ser manejados en programas multidisciplinarios y recibir educación en la enfermedad y las terapias, cuidados nutricionales y apoyo psicológico y social, entre otros ${ }^{10}$. En Colombia, tras la promulgación de la Declaración de Bogotá relativa a las iniciativas de salud renal, se han fomentado los programas de prevención de la ERC. El gobierno y las sociedades científicas han comenzado a apoyar estrategias de prevención de la ERC basadas en las directrices plasmadas en la declaración y resaltan la importancia para todos los grupos implicados de ofrecer una atención multidisciplinaria.

En este estudio describimos las características de una cohorte de pacientes con ERC que inician la TRR, se identifican los factores que influyen en el tiempo hasta la diálisis y se establecen las diferencias en este último desenlace de acuerdo al tipo de tratamiento recibido por el paciente, según sea éste cuidados multidisciplinarios, cuidados provistos únicamente por el nefrólogo o sin intervención.

\section{Materiales y métodos}

Se utilizó un diseño de cohorte retrospectiva que evaluó una población de pacientes elegibles de la red de clínicas renales de Renal Therapy Services (RTS) en Colombia. El periodo de reclutamiento fue del 1 de junio de 2008 al 31 de marzo de 2010. Se incluyeron pacientes con al menos 18 años de edad con ERC y una tasa de filtración glomerular estimada según el método de Cockcroft-Gault menor a $20 \mathrm{ml} / \mathrm{min}$ al comienzo del seguimiento. Fueron seguidos hasta su ingreso a diálisis, definido como el primer día de terapia dialítica crónica indicada por el médico tratante. Fueron excluidos todos aquellos pacientes con falla renal aguda o quienes requerían diálisis por rechazo al trasplante.

Las variables incluidas en el análisis fueron: edad, sexo, afiliación al sistema de salud, causa de la ERC, tasa de filtración glomerular y tensión arterial controlada, definida como una presión sanguínea sistólica $\leq 130 \mathrm{mmHg}$ y una presión sanguínea diastólica $\leq 90 \mathrm{mmHg}$. También se registraron variables de laboratorios clínicos: albúmina, calcio, fósforo, hemoglobina, 
glucosa y algunas variables del inicio de la TRR como el modo de inicio, el tipo de cuidado predialítico, el acceso vascular y la terapia al inicio. Adicionalmente, se evaluó el tipo de cuidados provistos a los pacientes antes del inicio de la TRR, y éstos fueron estratificados retrospectivamente en tres grupos de intervención: cuidados multidisciplinarios, cuidados únicamente por el nefrólogo o grupo de no intervención. Los pacientes del grupo de cuidados multidisciplinarios habían recibido un tratamiento de seguimiento durante un mínimo de tres meses y habían sido intervenidos de manera sistemática por un equipo de profesionales compuesto por nefrólogos, psicólogos, nutricionistas, enfermeras y trabajadores sociales; esta intervención hace énfasis en el logro de metas clínicas, soporte nutricional, educación previa al inicio de la terapia y soporte psicológico. Los pacientes del grupo de cuidados provistos únicamente por el nefrólogo no recibieron intervención por otros profesionales diferentes, habían recibido un tratamiento de seguimiento durante un mínimo de tres meses y realizaron al menos una visita a Nefrología en los 60 días previos al inicio de la terapia de diálisis, pero no participaban en ningún programa de intervención con cuidados multidisciplinarios. Los pacientes del grupo de no intervención no habían tenido contacto previo con un nefrólogo de la red de RTS antes de su ingreso en el registro.

En cuanto a la variable de desenlace ingreso a diálisis, se definió el ingreso programado como aquél que no requirió hospitalización para el inicio de la diálisis; por el contrario, el ingreso urgente fue todo aquél que sí la requirió.

Las variables clínicas y demográficas consideradas en el presente estudio se obtuvieron de los registros médicos electrónicos de RTS Red Nacional de Información Renal $\left(R_{E N I R}{ }^{\circledR}\right)$. Esta base de datos cuenta con la estructura y controles de calidad que garantizan la validez y confidencialidad de la información, y fue objeto de doble chequeo por los investigadores del estudio.

Para el análisis descriptivo se utilizaron proporciones para las variables categóricas y medias o medianas con sus respectivas medidas de dispersión para las variables continuas. Se usó el análisis de regresión de riesgos proporcionales de Cox con residuos de Schoenfeld para calcular los cocientes de riesgo (hazard ratio $[\mathrm{HR}])$. Estos residuos se emplearon para probar la proporcionalidad de los riesgos. Dado que la premisa de los riesgos proporcionales no era válida con algunas variables, se adaptaron modelos paramétricos alternativos. Se emplearon el criterio de información de Akaike (AIC) y el criterio de información bayesiano
(BIC) para comparar los diferentes modelos paramétricos. Los valores más bajos del AIC y del BIC se correspondieron con un modelo $\gamma$ generalizado (cuanto más bajos eran los valores del AIC y del BIC, mejor se ajustaba el modelo a los datos). Adicionalmente, con el fin de identificar los factores pronósticos relacionados con el inicio de la terapia (urgente frente a programada), se llevó a cabo un análisis de regresión logística. Con estos estimadores se empleó un intervalo de confianza (IC) del $95 \%$ y un valor de $p \leq 0.05$, los cuales fueron considerados estadísticamente significativos.

Adicionalmente se empleó el método de KaplanMeier para cuantificar el tiempo transcurrido desde el ingreso del paciente a la cohorte hasta el momento de inicio de la TRR. Se utilizó la prueba log-rank para comparar las funciones de supervivencia analizadas como tiempo libre de diálisis. El análisis estadístico se realizó en el software STATA 12 (StataCorp, College Station, Texas).

Este estudio fue aprobado y monitorizado por el Comité de Ética en Investigación de RTS (fecha del acta: 3 de enero de 2012).

\section{Resultados}

Un total de 769 pacientes cumplieron los criterios para el análisis. En la tabla 1 se muestran sus datos demográficos, clínicos y de laboratorio al comienzo del seguimiento, y en la tabla 2 se ofrecen las características de los pacientes al iniciar la TRR. El tiempo total desde el comienzo del seguimiento hasta la diálisis fue de 10,717.66 meses y se observaron 769 ingresos a diálisis, lo que equivale a una tasa de 7.18 inicios de TRR por cada 100 pacientes/mes. En la figura 1 se ofrece una curva de Kaplan-Meier que describe el tiempo hasta el ingreso a diálisis. Para todos los pacientes, la mediana de tiempo hasta el inicio de la TRR desde el comienzo del seguimiento fue de 5.2 meses (rango intercuartílico [IQR]: 15.9 meses); la mediana de tiempo hasta la diálisis en los pacientes que recibían cuidados multidisciplinarios fue de 14.9 meses (IQR: 15.9 meses); la mediana de tiempo en los pacientes que recibían intervención únicamente por parte del nefrólogo fue de 11.7 meses (IQR: 15.9 meses), y la mediana de tiempo en los pacientes que no recibían ninguna intervención fue de 0.1 meses (IQR: 0.34 meses). La comparación entre las funciones de supervivencia correspondientes a cada uno de los grupos se realizó con la prueba log-rank, y se encontró una diferencia significativa entre las funciones $\left(\chi^{2}(2)=22.63\right.$; $p<0.0001$ ). 
Tabla 1. Demografía y características desde el inicio del seguimiento

\begin{tabular}{|c|c|c|c|}
\hline Parámetros & $\begin{array}{l}\text { Cuidado multidisciplinario } \\
\qquad(\mathrm{n}=93)\end{array}$ & $\begin{array}{l}\text { Cuidado por Nefrología } \\
\qquad(\mathrm{n}=324)\end{array}$ & $\begin{array}{l}\text { Ninguna intervención } \\
\qquad(\mathrm{n}=352)\end{array}$ \\
\hline $\begin{array}{l}\text { Sexo, } \mathrm{n}(\%) \\
\text { Masculino } \\
\text { Femenino }\end{array}$ & $\begin{array}{l}37(39.8) \\
56(60.2)\end{array}$ & $\begin{array}{l}118(36.4) \\
206(63.6)\end{array}$ & $\begin{array}{l}157(44.6) \\
195(55.4)\end{array}$ \\
\hline Edad, media (DE) & $63.9(12.4)$ & $61.8(13.5)$ & $58.4(15.5)$ \\
\hline $\begin{array}{l}\text { Régimen de afiliación, n (\%) } \\
\text { Contributivo } \\
\text { Subsidiado } \\
\text { Vinculado }\end{array}$ & $\begin{array}{c}87(93.5) \\
6(6.5) \\
0\end{array}$ & $\begin{array}{l}283(87.4) \\
39(12.0) \\
2(0.6)\end{array}$ & $\begin{array}{l}214(60.8) \\
102(29.0) \\
36(10.2)\end{array}$ \\
\hline $\begin{array}{l}\text { Causa de la enfermedad renal, n (\%) } \\
\text { Diabetes } \\
\text { Hipertensión arterial } \\
\text { Glomerulonefritis } \\
\text { ERC obstructiva } \\
\text { Enfermedad renal poliquística } \\
\text { Otra } \\
\text { Desconocida }\end{array}$ & $\begin{array}{l}55(59.1) \\
14(15.1) \\
14(15.1) \\
0 \\
0 \\
1(1.1) \\
9(9.6)\end{array}$ & $\begin{array}{l}181(55.9) \\
62(19.1) \\
20(6.2) \\
7(2.1) \\
10(3.1) \\
23(7.1) \\
21(6.5)\end{array}$ & $\begin{array}{l}141(40.1) \\
97(27.6) \\
31(8.8) \\
18(5.1) \\
9(2.5) \\
19(5.4) \\
37(10.5)\end{array}$ \\
\hline Presión arterial controlada, n (\%) & $41(44.1)$ & $107(33.0)$ & $111(31.5)$ \\
\hline $\begin{array}{l}\text { Laboratorios séricos, media (DE) } \\
\text { Albúmina, } \mathrm{g} / \mathrm{dl} \\
\text { Calcio, } \mathrm{mmol} / \mathrm{l} \\
\text { Glucosa, } \mathrm{mmol} / \mathrm{l} \\
\text { Hemoglobina, } \mathrm{g} / \mathrm{dl} \\
\text { Fósforo, } \mathrm{mmol} / \mathrm{l}\end{array}$ & $\begin{array}{c}3.6(0.5) \\
8.4(1.1) \\
123.6(58.8) \\
10.6(1.5) \\
5.3(1.3)\end{array}$ & $\begin{array}{c}3.6(0.6) \\
8.6(1.3) \\
132.6(60.3) \\
10.1(1.6) \\
4.9(1.3)\end{array}$ & $\begin{array}{c}3.5(0.7) \\
8.5(1.3) \\
129.2(63.7) \\
9.3(1.7) \\
4.9(1.6)\end{array}$ \\
\hline $\begin{array}{l}\text { Media de la tasa de filtración glomerular, } \\
\mathrm{ml} / \mathrm{min} / 1.73 \mathrm{~m}^{2}\end{array}$ & $12.7(3.5)$ & $12.4(4.1)$ & $9.9(3.6)$ \\
\hline
\end{tabular}

DE: desviación estándar.

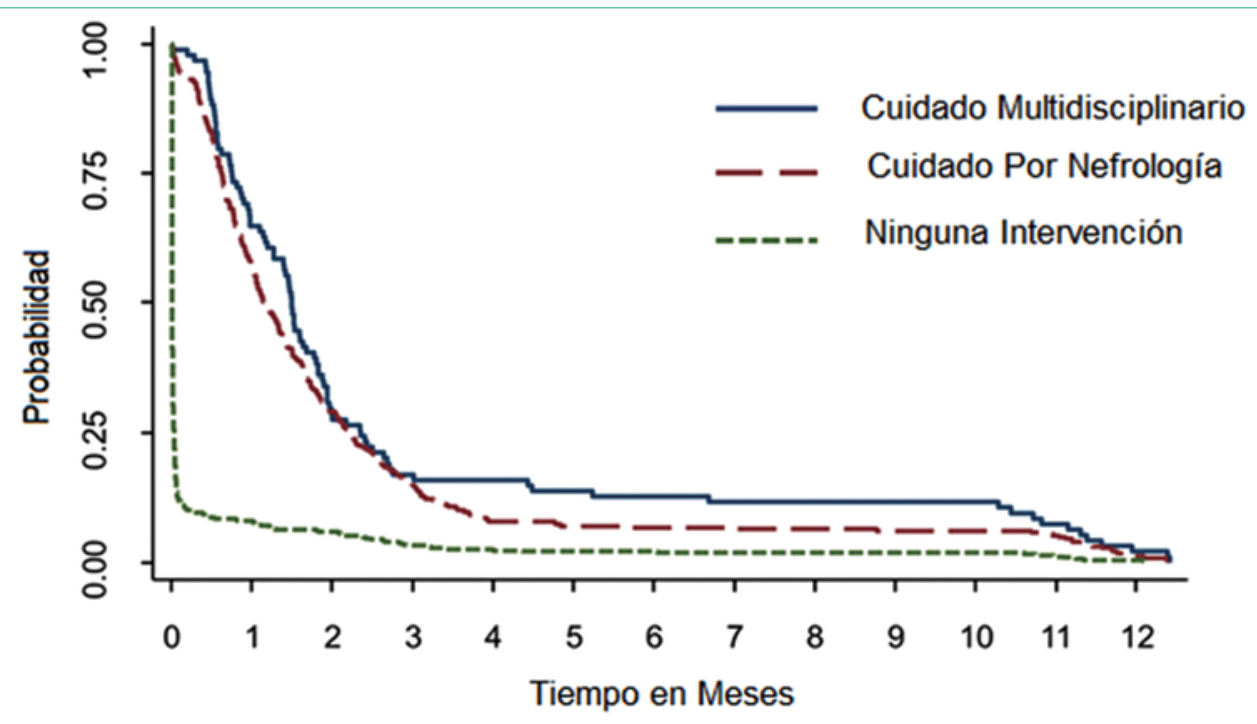

Figura 1. Tiempo hasta el inicio de la terapia de reemplazo renal (TRR).

Una estimación paramétrica del tiempo hasta el En la tabla 3 se muestran los HR del inicio de la ingreso a diálisis con un modelo $\gamma$ generalizado cal- TRR asociados con cada uno de los factores de culó los HR crudos y ajustados del inicio de la TRR. riesgo. 
Nefro Lat. 2018;15

Tabla 2. Características de los pacientes al inicio de la TRR

\begin{tabular}{|c|c|c|c|}
\hline Características & $\begin{array}{l}\text { Cuidado multidisciplinario } \\
\qquad(\mathrm{n}=93)\end{array}$ & $\begin{array}{l}\text { Cuidado por Nefrología } \\
\qquad(\mathrm{n}=324)\end{array}$ & $\begin{array}{l}\text { Ninguna intervención } \\
\qquad(\mathrm{n}=352)\end{array}$ \\
\hline $\begin{array}{l}\text { Modo de inicio, n (\%) } \\
\text { Programado } \\
\text { Urgencia } \\
\text { Sin dato }\end{array}$ & $\begin{array}{c}71(76.3) \\
22(23.7) \\
0\end{array}$ & $\begin{array}{c}213(65.7) \\
111(34.3) \\
0\end{array}$ & $\begin{array}{c}157(44.6) \\
193(54.8) \\
2(0.6)\end{array}$ \\
\hline $\begin{array}{l}\text { Acceso de inicio de terapia } \\
\text { Catéter peritoneal } \\
\text { Catéter vascular } \\
\text { Fístula arteriovenosa }\end{array}$ & $\begin{array}{c}59(63.4) \\
31(33.3) \\
3(3.2)\end{array}$ & $\begin{array}{c}190(58.6) \\
124(38.3) \\
10(3.1)\end{array}$ & $\begin{array}{c}145(41.2) \\
199(56.5) \\
8(2.3)\end{array}$ \\
\hline $\begin{array}{l}\text { Inicio de TRR, n (\%) } \\
\text { DP continua ambulatoria } \\
\text { DP automatizada } \\
\text { Hemodiálisis }\end{array}$ & $\begin{array}{l}30(32.3) \\
29(31.2) \\
34(36.6)\end{array}$ & $\begin{array}{l}128(39.5) \\
62(19.1) \\
134(41.4)\end{array}$ & $\begin{array}{l}101(28.7) \\
44(12.5) \\
207(58.8)\end{array}$ \\
\hline
\end{tabular}

En el análisis univariado, las variables asociadas con una duración significativamente más larga del seguimiento hasta el inicio de la TRR fueron las siguientes: ser varón ( $p=0.033)$, mantener el control de la presión sanguínea $(p=0.006)$, ser atendido por un equipo multidisciplinario o un nefrólogo $(p<0.001)$ y presentar niveles de hemoglobina $(p<0.001)$ y de albúmina $(p=0.033)$ dentro del rango normal. En cambio, las variables asociadas con una duración significativamente más breve del seguimiento hasta el inicio de la TRR fueron: ser menor de 60 años $(p<0.05)$, contar con un plan subsidiado de salud o no estar cubierto en absoluto por la seguridad social $(p<0.001)$ y que la causa de la ERC fuera una enfermedad urinaria obstructiva ( $p$ < 0.001). En el análisis multivariado (Tabla 3), las variables relacionadas con un tiempo significativamente más corto hasta la TRR fueron las siguientes: no estar cubierto por la seguridad social $(p<0.001)$, presentar ERC debida a una causa obstructiva $(p<0.001)$ o desconocida $(p=0.015)$, no haber recibido ningún cuidado ( $p<0.001)$ y mostrar un escaso control glucémico $(p<0.022)$. El hecho de tener la presión sanguínea controlada se asoció con un tiempo significativamente más largo hasta la TRR ( $p=0.018)$.

Empleando un modelo de regresión logística, y ajustándolo para considerar otras variables, buscamos identificar aquellas variables que repercutían en el tipo de ingreso a la terapia (urgente frente a programada) (Tabla 4). Las variables asociadas con un inicio urgente de la TRR fueron: ser hombre, no recibir cuidados de un equipo multidisciplinario ni de un nefrólogo y presentar niveles de hemoglobina fuera del rango normal.

\section{Discusión}

La evidencia actual demuestra una mayor tasa de morbilidad y mortalidad en aquellos pacientes de diálisis que han sido remitidos tardíamente a cualquier cuidado predialítico (definido, por lo general, como cuatro meses o menos desde la necesidad de diálisis) ${ }^{4,11,12}$. En el presente estudio la mediana de tiempo hasta el inicio de la TRR desde el comienzo del seguimiento fue de 5.2 meses. Este plazo podría considerarse corto con los cuidados predialíticos adecuados y representaría una utilización subóptima de los programas de prevención del sistema colombiano de salud y una falta de acceso de los pacientes a los cuidados predialíticos. El criterio de inclusión de una tasa de filtración glomerular menor a $20 \mathrm{ml} / \mathrm{min}$ redujo el periodo de seguimiento, lo que también explica el breve periodo de tiempo hasta la TRR, en especial en el grupo de no intervención.

Los resultados de nuestro análisis lineal generalizado $\gamma$ demuestran una fuerte asociación entre el periodo de tiempo libre de diálisis y no estar cubierto por la seguridad social. La ausencia de afiliación a la seguridad social refleja desigualdades económicas y sociales e influye en el acceso y el resultado clínico. Algunos estudios han demostrado que una baja posición socioeconómica y menores niveles educativos están asociados con una remisión tardía a los cuidados nefrológicos ${ }^{13}$. Asimismo, nuestro análisis demuestra que el hecho de que la ERC sea de origen obstructivo 0 desconocido indica también un corto plazo hasta la diálisis. Este último hallazgo llama mucho la atención, sobre todo si se tiene en cuenta que un subgrupo importante de las causas obstructivas puede ser exitosamente intervenido con procesos derivativos que alivian y/o protegen la función del riñón. Estos predictores pueden explicarse mediante una progresión no lineal de la enfermedad y la rápida aparición de la enfermedad renal en estadio terminal provocada por eventos agudos, según describe Onuigbo ${ }^{14}$. Por otro lado, los resultados de este estudio demuestran que entre los 
Tabla 3. Análisis $\gamma$ generalizado para los factores de riesgo en el tiempo transcurrido de TRR

\begin{tabular}{|c|c|c|c|c|c|}
\hline \multirow[t]{2}{*}{ Parámetros } & \multirow[t]{2}{*}{ Categoría } & \multicolumn{2}{|c|}{ Univariado } & \multicolumn{2}{|c|}{ Multivariado } \\
\hline & & HR (IC 95\%) & Valor de p & HR (IC 95\%) & Valor de $p$ \\
\hline Edad al inicio de la TRR & $\begin{array}{l}0-49 \\
50-59 \\
60-69 \\
\geq 70\end{array}$ & $\begin{array}{c}1.52(1.24-1.87) \\
1.24(1.02-1.51) \\
1.10(0.91-1.34) \\
1 \text { (ref.) }\end{array}$ & $\begin{array}{l}0.000 \\
0.034 \\
0.311\end{array}$ & $\begin{array}{l}1.08(0.75-1.54) \\
0.93(0.73-1.19) \\
0.99(0.78-1.24)\end{array}$ & $\begin{array}{l}0.601 \\
0.554 \\
0.911\end{array}$ \\
\hline Sexo & $\begin{array}{l}\text { Masculino } \\
\text { Femenino }\end{array}$ & $\begin{array}{c}0.86(0.74-0.98) \\
1 \text { (ref.) }\end{array}$ & 0.033 & $0.91(0.76-1.08)$ & 0.269 \\
\hline Régimen de afiliación & $\begin{array}{l}\text { Subsidiado } \\
\text { No asegurado } \\
\text { Contributivo }\end{array}$ & $\begin{array}{c}2.04(1.7-2.45) \\
5.97(4.24-8.41) \\
1 \text { (ref.) }\end{array}$ & $\begin{array}{l}0.000 \\
0.000\end{array}$ & $\begin{array}{l}1.16(0.92-1.48) \\
2.55(1.67-3.89)\end{array}$ & $\begin{array}{l}0.212 \\
0.000\end{array}$ \\
\hline $\begin{array}{l}\text { Causa de la enfermedad } \\
\text { renal }\end{array}$ & $\begin{array}{l}\text { Desconocida } \\
\text { Diabetes } \\
\text { Enfermedad renal poliquística } \\
\text { Glomerulonefritis } \\
\text { ERC obstructiva } \\
\text { Otra } \\
\text { Hipertensión arterial }\end{array}$ & $\begin{array}{c}1.29(0.97-1.70) \\
0.86(0.72-1.03) \\
0.90(0.56-1.43) \\
0.85(0.64-1.13) \\
3.06(2.00-4.67) \\
0.80(0.57-1.11) \\
1 \text { (ref.) }\end{array}$ & $\begin{array}{l}0.079 \\
0.093 \\
0.647 \\
0.271 \\
0.000 \\
0.184\end{array}$ & $\begin{array}{l}1.56(1.09-2.25) \\
0.95(0.75-1.21) \\
1.07(0.62-1.85) \\
0.85(0.59-0.47) \\
3.81(2.20-6.60) \\
0.70(0.45-1.09)\end{array}$ & $\begin{array}{l}0.015 \\
0.700 \\
0.794 \\
0.426 \\
0.000 \\
0.119\end{array}$ \\
\hline $\begin{array}{l}\text { Control de la presión } \\
\text { arterial }\end{array}$ & $\begin{array}{l}\text { Controlada } \\
\text { No controlada }\end{array}$ & $\begin{array}{c}0.81(0.70-0.94) \\
1 \text { (ref.) }\end{array}$ & 0.006 & $0.80(0.67-0.96)$ & 0.018 \\
\hline Grupo de intervención & $\begin{array}{l}\text { Cuidado multidisciplinario } \\
\text { Cuidado sólo por Nefrología } \\
\text { Ninguna intervención }\end{array}$ & $\begin{array}{c}0.21(0.17-0.27) \\
0.25(0.21-0.29) \\
1 \text { (ref.) }\end{array}$ & $\begin{array}{l}0.000 \\
0.000\end{array}$ & $\begin{array}{l}0.20(0.15-0.27) \\
0.23(0.19-0.28)\end{array}$ & $\begin{array}{l}0.000 \\
0.000\end{array}$ \\
\hline $\begin{array}{l}\text { Prueba de laboratorios } \\
\text { séricos }\end{array}$ & $\begin{array}{l}\text { Hemoglobina } \\
\text { Albúmina } \\
\text { Fósforo } \\
\text { Glucosa }\end{array}$ & $\begin{array}{l}0.87(0.83-0.90) \\
0.87(0.76-0.99) \\
1.03(0.98-1.08) \\
1.00(0.99-1.00)\end{array}$ & $\begin{array}{l}0.000 \\
0.033 \\
0.244 \\
0.383\end{array}$ & $\begin{array}{l}0.97(0.92-1.03) \\
0.87(0.75-1.01) \\
1.03(0.98-1.10) \\
1.00(0.99-1.01)\end{array}$ & $\begin{array}{l}0.318 \\
0.074 \\
0.240 \\
0.022\end{array}$ \\
\hline
\end{tabular}

pronosticadores de un mayor tiempo hasta la diálisis se incluye ser varón, tener controlada la presión sanguínea y contar con niveles normales de albúmina y hemoglobina. Los pacientes que reciben cuidados multidisciplinarios 0 , al menos, solamente del nefrólogo son más propensos a presentar valores de química sanguínea que cumplen con los parámetros clínicos y de laboratorio controlados, lo que se correlaciona con una lenta progresión de la $\mathrm{ERC}^{15,16}$.

El inicio planeado de la diálisis es un importante indicador de la calidad de los cuidados predialíticos. Entre los pacientes incluidos en este estudio, el $42.4 \%$ tuvo un inicio urgente de la diálisis, lo que demuestra la necesidad de mejorar el acceso de los pacientes a los cuidados predialíticos en Colombia. Los hombres fueron más significativamente propensos a un inicio urgente. En Colombia, los hombres suelen ser menos precavidos y postergan el acceso a los servicios de salud $^{17}$. Este estudio también demostró que los pacientes a quienes se les administró algún cuidado predialítico tuvieron un menor riesgo de inicio urgente ${ }^{16,18,19}$, debido en parte a un mejor control de sus niveles de hemoglobina y albúmina, un factor previamente documentado como ayuda para prevenir el inicio urgente de la diálisis ${ }^{20}$. Además, se observa una tendencia de que los pacientes provenientes de cuidado pre dialítico o atención por el nefrólogo tienen una mayor proporción de inicio por diálisis peritoneal (DP). Este estudio también se hace eco de la muy escasa proporción de pacientes que inicia la hemodiálisis con una fístula arteriovenosa (2.8\%), a pesar de que es ampliamente conocido que los pacientes con catéteres vasculares se enfrentan a un mayor riesgo de peores desenlaces en diálisis ${ }^{21}$.

Una limitación de este estudio es la desproporción en el número de pacientes entre los grupos: hubo casi cuatro veces más en los grupos de no intervención y de cuidados únicamente por el nefrólogo que en el grupo de cuidados multidisciplinarios. No obstante, dado que esta cohorte de pacientes recibe tratamiento en una red de instalaciones renales con centros ubicados en todo el país, se prevé que represente las características de los cuidados predialíticos en toda Colombia. Por consiguiente, nuestros resultados podrían emplearse para alertar a los responsables de la toma de decisiones en materia de salud y animarlos a 
Nefro Lat. 2018;15

Tabla 4. Modelo logístico, modo de inicio de la TRR (de urgencia vs. programado)

\begin{tabular}{|c|c|c|c|}
\hline Parámetros & Categoría & Inicio urgente OR (IC 95\%) & Valor de $p$ \\
\hline Edad al inicio de la TRR & $\begin{array}{l}0-49 \\
50-59 \\
60-69 \\
\geq 70\end{array}$ & $\begin{array}{c}0.998(0.551-1.809) \\
1.218(0.719-2.064) \\
1.097(0.657-1.830) \\
1 \text { (ref.) }\end{array}$ & $\begin{array}{l}0.995 \\
0.464 \\
0.723\end{array}$ \\
\hline Sexo & $\begin{array}{l}\text { Masculino } \\
\text { Femenino }\end{array}$ & $\begin{array}{c}1.549 \text { (1.052-2.282) } \\
1 \text { (ref.) }\end{array}$ & 0.027 \\
\hline Régimen de afiliación & $\begin{array}{l}\text { Subsidiado } \\
\text { No asegurado } \\
\text { Contributivo }\end{array}$ & $\begin{array}{c}1.092(0.655-1.822) \\
1.944(0.788-4.793) \\
1 \text { (ref.) }\end{array}$ & $\begin{array}{l}0.736 \\
0.149\end{array}$ \\
\hline Causa de la enfermedad renal & $\begin{array}{l}\text { Desconocida } \\
\text { Diabetes } \\
\text { Enfermedad renal poliquística } \\
\text { Glomerulonefritis } \\
\text { ERC obstructiva } \\
\text { Otra } \\
\text { Hipertensión arterial }\end{array}$ & $\begin{array}{c}1.155(0.537-2.484) \\
0.830(0.490-1.405) \\
2.026(0.636-6.449) \\
1.861(0.827-4.188) \\
2.893(0.813-10.289) \\
0.876(0.343-2.236) \\
1 \text { (ref.) }\end{array}$ & $\begin{array}{l}0.712 \\
0.488 \\
0.232 \\
0.133 \\
0.101 \\
0.109\end{array}$ \\
\hline Control de la presión arterial & $\begin{array}{l}\text { Controlada } \\
\text { No controlada }\end{array}$ & $\begin{array}{c}0.813 \text { (0.546-1.209) } \\
1 \text { (ref.) }\end{array}$ & 0.782 \\
\hline Grupo de intervención & $\begin{array}{l}\text { Cuidado multidisciplinario } \\
\text { Cuidado por Nefrología } \\
\text { Sin intervención }\end{array}$ & $\begin{array}{c}0.400(0.213-0.752) \\
0.464(0.307-0.703) \\
1 \text { (ref.) }\end{array}$ & $\begin{array}{l}0.004 \\
0.000\end{array}$ \\
\hline Prueba de laboratorios séricos* & $\begin{array}{l}\text { Hemoglobina } \\
\text { Albúmina } \\
\text { Fósforo } \\
\text { Glucosa }\end{array}$ & $\begin{array}{l}0.766(0.675-0.868) \\
0.800(0.583-1.097) \\
0.911(0.798-1.041) \\
1.002(0.998-1.005)\end{array}$ & $\begin{array}{l}0.000 \\
0.166 \\
0.171 \\
0.314\end{array}$ \\
\hline
\end{tabular}

TRR: terapia de reemplazo renal; OR: odds ratio; IC: intervalo de confianza; ERC: enfermedad renal crónica

mejorar los programas predialíticos, con el fin de lograr mejores resultados y el acceso a los cuidados predialíticos de los pacientes con ERC.

\section{Conclusiones}

El cuidado provisto por un equipo multidisciplinario o por el nefrólogo aumenta el tiempo libre de diálisis en pacientes con ERC avanzada.

\section{Responsabilidades éticas}

Protección de personas y animales. Los autores declaran que para esta investigación no se han realizado experimentos en seres humanos ni en animales.

Confidencialidad de los datos. Los autores declaran que han seguido los protocolos de su centro de trabajo sobre la publicación de datos de pacientes.

Derecho a la privacidad y consentimiento informado. Los autores declaran que en este artículo no aparecen datos de pacientes.

\section{Conflicto de intereses}

Ángela S. Rivera es empleada a tiempo completo de Baxter Latinoamérica. Delia Perea, Kindar R. Astudillo, J. Mauricio Uribe, Izcay Ronderos, Luis Fernando Moreno, Alfonso Bunch, Leyder Corzo y Mauricio Sanabria son empleados a tiempo completo de RTS, una filial de Baxter Colombia. Ricardo Sánchez mantiene una relación como asesor con Baxter Colombia. J. Mauricio Uribe mantiene una relacion con la Universidad Pontificia Bolivariana.

\section{Agradecimientos}

El apoyo financiero para la asistencia editorial ha sido proporcionado por Baxter. Los autores quieren expresar su agradecimiento a Lamara D. Shrode, PhD, profesional de publicaciones médicas certificada por la ISMPPTM de The JB Ashtin Group, Inc., quien ha ofrecido su asistencia editorial y realizado modificaciones a la obra de los autores en nombre de Baxter. 


\section{Bibliografía}

1. Coresh J, Selvin E, Stevens LA, Manzi J, Kusek JW, Eggers P, et al. Prevalence of chronic kidney disease in the United States. JAMA. 2007;298(17):2038-47.

2. Levey AS, Atkins R, Coresh J, Cohen EP, Collins AJ, Eckardt KU, et al. Chronic kidney disease as a global public health problem: approaches and initiatives - a position statement from Kidney Disease Improving Global Outcomes. Kidney Int. 2007;72(3):247-59.

3. Schieppati A, Remuzzi G. Chronic renal diseases as a public health problem: epidemiology, social, and economic implications. Kidney Int Suppl. 2005;(98):S7-S10.

4. Huisman RM. The deadly risk of late referral. Nephrol Dial Transplant. 2004;19(9):2175-80.

5. Hemmelgarn BR, Manns BJ, Zhang J, Tonelli M, Klarenbach S, Walsh M, Culleton BF. Association between multidisciplinary care and survival for elderly patients with chronic kidney disease. J Am Soc Nephrol. 2007; 18(3):993-9.

6. Kazmi WH, Obrador GT, Khan SS, Pereira BJ, Kausz AT. Late nephrology referral and mortality among patients with end-stage renal disease: a propensity score analysis. Nephrol Dial Transplant. 2004; 19(7):1808-14.

7. Mendelssohn DC. Coping with the CKD epidemic: the promise of multidisciplinary team-based care. Nephrol Dial Transplant. 2005;20(1):10-2.

8. Khan SS, Xue JL, Kazmi WH, Gilbertson DT, Obrador GT, Pereira BJ, et al. Does predialysis nephrology care influence patient survival after initiation of dialysis? Kidney Int. 2005:67(3):1038-46.

9. Bayliss EA, Bhardwaja B, Ross C, Beck A, Lanese DM. Multidisciplinary team care may slow the rate of decline in renal function. Clin J Am Soc Nephrol. 2011;6(4):704-10.

10. Levin A, Stevens PE, Bilous RW, Coresh J, De Francisco AL, De Jong PE, et al. Improving Global Outcomes (KDIGO) CKD Work Group. KDIGO 2012 clinical practice guideline for the evaluation and management of chronic kidney disease. Kidney Int Suppl. 2013;3(1):1-150.
11. Stack AG. Impact of timing of nephrology referral and pre-ESRD care on mortality risk among new ESRD patients in the United States. Am J Kidney Dis. 2003;41(2):310-8.

12. Winkelmayer WC, Owen WF, Jr., Levin R, Avorn J. A propensity analysis of late versus early nephrologist referral and mortality on dialysis. J Am Soc Nephrol. 2003;14(2):486-92.

13. Navaneethan SD, Aloudat $S$, Singh $S$. A systematic review of patient and health system characteristics associated with late referral in chronic kidney disease. BMC Nephrol. 2008;9:3.

14. Onuigbo MA. Syndrome of rapid-onset end-stage renal disease: a new unrecognized pattern of CKD progression to ESRD. Ren Fail. 2010; 32(8):954-8

15. Dixon J, Borden P, Kaneko TM, Schoolwerth AC. Multidisciplinary CKD care enhances outcomes at dialysis initiation. Nephrol Nurs J. 2011; 38(2):165-71.

16. Goldstein M, Yassa T, Dacouris N, McFarlane P. Multidisciplinary cuidado predialítico and morbidity and mortality of patients on dialysis. Am J Kidney Dis. 2004;44(4):706-14.

17. Encuesta Nacional de Salud Pública. Ministerio de la Protección Social Colombia. [Internet] Consultado el 25 de febrero de 2015. Disponible en: http:// www.minsalud.gov.co/salud/Paginas/EncuestaNacionaldeSaludPublica.aspx.

18. Levin A, Lewis M, Mortiboy P, Faber S, Hare I, Porter EC, et al. Multidisciplinary predialysis programs: quantification and limitations of their impact on patient outcomes in two Canadian settings. Am J Kidney Dis. 1997;29(4):533-40.

19. Yeoh HH, Tiquia HS, Abcar AC, Rasgon SA, Idroos ML, Daneshvari SF. Impact of cuidado predialítico on clinical outcomes. Hemodial Int. 2003;7(4):338-41.

20. Curtis BM, Ravani P, Malberti F, Kennett F, Taylor PA, Djurdjev O, et al. The short- and long-term impact of multi-disciplinary clinics in addition to standard nephrology care on patient outcomes. Nephrol Dial Transplant. 2005;20(1):147-54

21. Perl J, Wald R, McFarlane P, Bargman JM, Vonesh E, Na Y, et al. Hemodialysis vascular access modifies the association between dialysis modality and survival. J Am Soc Nephrol. 2011;22(6):1113-21. 